\title{
Revealing the Metabolic Profile of Brain Tumors for Diagnosis Purposes
}

\author{
M. G. Kounelakis, M. E. Zervakis, G. J. Postma, L. M. C. Buydens, A. Heerschap and X. Kotsiakis
}

\begin{abstract}
The metabolic behavior of complex brain tumors, like Gliomas and Meningiomas, with respect to their type and grade was investigated in this paper. Towards this direction the smallest set of the most representative metabolic markers for each brain tumor type was identified, using ratios of peak areas of well established metabolites, from ${ }^{1} \mathrm{H}$-MRSI (Proton Magnetic Resonance Spectroscopy Imaging) data of 24 patients and 4 healthy volunteers. A feature selection method that embeds Fisher's filter criterion into a wrapper selection scheme was applied; Support Vector Machine (SVM) and Least Squares-SVM (LS-SVM) classifiers were used to evaluate the ratio markers classification significance. The area under the Receiver Operating Characteristic curve (AUROC) was adopted to evaluate the classification significance. It is found that the NAA/CHO, $\mathrm{CHO} / \mathrm{S}, \mathrm{MI} / \mathrm{S}$ ratios can be used to discriminate Gliomas and Meningiomas from Healthy tissue with AUROC greater than 0.98. Ratios $\mathrm{CHO} / \mathrm{S}, \mathrm{CRE} / \mathrm{S}, \mathrm{MI} / \mathrm{S}$, LAC/CRE, ALA/CRE, ALA/S and LIPS/CRE can identify type and grade differences in Gliomas giving AUROC greater than 0.98 apart from the scheme of Gliomas grade II vs grade III where 0.84 was recorded due to high heterogeneity. Finally NAA/CRE, NAA/S, CHO/S, MI/S and ALA/S manage to discriminate Gliomas from Meningiomas providing AUROC exceeding 0.90 .
\end{abstract}

\section{INTRODUCTION}

$\mathrm{B}$ RAIN tumor diagnosis often requires a resection of brain's tissue in order to study in depth the type and grade of the tumor, as to proceed with the proper therapy. Such an invasive technique involves the cost of losing vital brain functions of the patient. Therefore, reliable but noninvasive techniques are required to eliminate, if possible, this risk.

One of the most promising technologies towards this direction is the ${ }^{1} \mathrm{H}-\mathrm{MRSI}$ (Proton Magnetic Resonance Spectroscopy Imaging) which provides metabolic profile of the tumors through the spectra acquired from small cubic volume of interests (VOI) called voxels, as in Fig $1 .{ }^{1} \mathrm{H}-$ MRSI technology has been used in brain tumors diagnosis over the last decade [1]. Using these spectra, the concentration of known metabolites can be measured and

Manuscript submitted June 18, 2009

M. G. Kounelakis and M. E. Zervakis are with the Technical University of Crete, Department of Electronic and Computer Engineering.

G. J. Postma and L. M. C. Buydens are with the Radboud University, Department of Analytical Chemistry.

A. Heerschap, is with the University Medical Center of Nijmegen, Department of Radiology.

$\mathrm{X}$. Kotsiakis, is with the General Hospital of Chania, Department of Neurosurgery. then extract the necessary metabolic information of the tumors. Further description of these metabolites is provided in the next section.

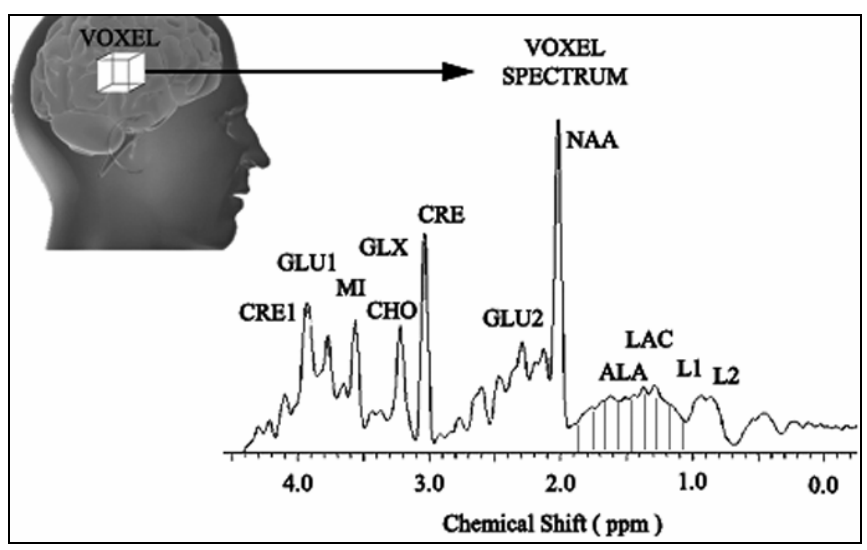

Fig. 1. The metabolite spectrum generated from a brain voxel. Y axis: peak heights (metabolites concentration). $\mathrm{X}$ axis: frequency (position) in parts per million. The lined area shows the area under the metabolite's peak.

The aim of this study is to employ the metabolic information extracted from 24 patients and 4 healthy volunteers (569 voxels) in order to identify the smallest set of the most significant metabolic markers that could be used to accurately describe the type and grade of the tumors, thus assisting the diagnosis practice. For this purpose, ratios of the areas under the metabolites peaks are used. Measuring the metabolites' peak area ratios has the advantage of canceling out the effects of general reduction in measured metabolite concentrations, which are due to variations in cellular density.

Ratios of metabolites peak areas have been already used in the classification of several types of tumors [2], [3]. Most of previous studies, however, only deal with the ratios of two or three known metabolites such as NAA (N-acetylaspartate, CRE (Creatine) and CHO (Choline). The novelty here is that 31 different peak area ratios are constructed and tested from 12 known metabolites indicated in Fig. 1. The process of feature selection with the subsequent evaluation of the classification accuracy forms a subjective way of selecting the most effective subset of such ratios.

\section{MATERIALS AND METHODS}

\section{A. Materials}

The core dataset consists of 569 pre-processed spectral sets containing MRS data from 24 brain tumor patients and 
4 control persons. More specifically, there are 10 Gliomas patients of Grade II, 4 of Grade III, 7 of Grade IV and 3 Meningiomas patients. Each patient passed strict quality control and validation procedures, including consensus histopathologic determination. Per tissue type voxels were taken from homogeneous regions. The Healthy tissue from patients was collected from the contralateral half of the brain.

The areas under the metabolites' peaks were obtained by peak integration [4]. More specifically, these areas were estimated by integrating the spectral intensities of each metabolite within a window of $0.13 \mathrm{ppm}$, Fig. 1 , in the range 0.5 to $4.0 \mathrm{ppm}$. This range is the most suitable to obtain secure measurements since the metabolites' peaks can be easily measured. Metabolites of particular interest include total Creatine (CRE1 with peak at $3.95 \mathrm{ppm}$ ), Glutamate (GLU1 with peak at $3.75 \mathrm{ppm}$ ), Myo-inositol (MI with peak at $3.56 \mathrm{ppm}$ ), Glutamate/Glutamine (GLX with peak at 3.44 $\mathrm{ppm})$, Choline ( $\mathrm{CHO}$ with peak at $3.20 \mathrm{ppm})$, Creatine (CRE with peak at $3.02 \mathrm{ppm}$ ), Glutamine (GLU2 with peak at $2.20 \mathrm{ppm}$ ), N-acetyl aspartate (NAA with peak at 2.02 $\mathrm{ppm}$ ), Alanine (ALA with peak at $1.48 \mathrm{ppm}$ ), Lactate (LAC with peak at $1.33 \mathrm{ppm})$, Lipids1 (L1 with peak at $1.30 \mathrm{ppm}$ ) and Lipids2 (L2 with peak at $0.90 \mathrm{ppm}$ ). The $0.13 \mathrm{ppm}$ window was selected as a good integration area covering the range of most peaks of interest, without being contaminated with neighboring peaks. The selected metabolites have also a biological relation in tumor formation [5].

The classification schemes designed were the following: Healthy vs Tumors, Healthy vs Gliomas, Healthy vs Meningiomas, Gliomas Grade II (GR2) vs Gliomas Grade III (GR3), Gliomas Grade II vs Gliomas Grade IV (GR4), Gliomas Grade III vs Gliomas Grade IV (Glioblastomas), Gliomas Grade II vs Meningiomas (MNG), Gliomas Grade III vs Meningiomas and Gliomas Grade IV vs Meningiomas.

\section{B. Feature Selection \& Classification Method}

In order to identify the most significant metabolic markers that could suggest an alternative, noninvasive diagnosis protocol, a feature selection and classification method was adopted, which has been previously used on genomic data with remarkable results [6]. A feature set of 31 ratios of metabolites' peak areas was entered to the algorithm in order to select, through a recursive feature elimination process, the smallest set of the most significant ratio markers, for each brain tissue type. Subsequently, the discrimination power of these markers was evaluated through the 9 different binary classification schemes, using Support Vector Machines (SVMs) and Least Square-Support Vector Machines (LSSVMs) classifiers with a Radial Basis Function (RBF) kernel [7], [8]. Kernel-based methods can perform well in processing when high dimensional and heterogeneous data are under scrutiny. Even more, these methods are able to cope with possible discrepancies of feature values since they map them, through the kernel technique, to another space called feature space where linear discrimination can be applied. These facts motivated the inclusion of SVM and LS-SVM in this study.

Another important characteristic of this method is that the Fisher's criterion in (1) is embedded in the feature selection process in order to rank the ratio features. This criterion is applied only to the Support Vectors of the two classes (positive and negative), since these form the most representative vectors for classification. Through the selection scheme, the least significant ratio feature, i.e. the ratio feature with the smallest Fisher's value is discarded after each iteration and the most significant ones are kept for further classification purposes. This process is repeated until the best classification accuracy reached with the smallest possible number of ratio features. In this way the smallest set of the most significant ratio markers is selected for each classification scheme and presented in the next section, in Table I. The Fisher's criterion has the form

$$
f\left(x_{i}\right)=\frac{\left|\mu_{+}\left(x_{i}\right)-\mu_{-}\left(x_{i}\right)\right|}{\sigma_{+}\left(x_{i}\right)+\sigma_{-}\left(x_{i}\right)}
$$

where $\mu_{+}\left(x_{i}\right)-\mu_{-}\left(x_{i}\right)$ and $\sigma_{+}\left(x_{i}\right)+\sigma_{-}\left(x_{i}\right)$ are the mean and standard deviation values of feature $x_{i}$ in the positive and negative classes, respectively.

The evaluation of classifier performance was assessed through two different strategies, namely 10 -fold Cross Validation (CV) and Leave-One-Patient-Out CV. Classification accuracy was measured in terms of AUROC values, along with the associated Confidence Intervals (CI) which indicates the expected deviation of accuracy and reflects the generalization ability of the classification scheme.

Statistical significance of the selected ratio markers was also measured through Independent-Samples T test method. The means of the ratio markers were estimated in both classes and the p-values were computed. A ratio marker was considered significant when its p-value was less than 0.05 and highly significant for less that 0.001 .

\section{EXPERIMENTAL RESULTS}

The experimental results are presented in Tables I and II, regarding the selected markers and their significance, respectively. More specifically, the smallest sets of significant features achieving the best AUROC measure are indicated in Table I for the classification problems considered. Furthermore, Table II illustrates schematically the significance of features where a single arrow denotes a significant ratio marker, while double arrow corresponds to a highly significant ratio marker. The upwards direction of the arrow reflects an increase of the ratio's mean in the second class while downwards implies a decrease. 
TABLE I

MOST SIGNIFICANT FEATURES FOR EACH BINARY CLASSIFICATION SCHEME

\begin{tabular}{|c|c|c|c|c|c|c|c|c|c|}
\hline \multirow{2}{*}{$\begin{array}{c}\begin{array}{c}\text { Binary } \\
\text { schemes }\end{array} \\
\text { Healthy vs } \\
\text { Tumors }\end{array}$} & \multicolumn{8}{|c|}{ Most frequent features at best AUROC } & \multirow{2}{*}{$\begin{array}{c}\text { Best } \\
\text { AUROC / CI } \\
0.99 / 0.004\end{array}$} \\
\hline & $\mathrm{NAA} / \mathrm{CHO}$ & $\mathrm{CHO} / \mathrm{CRE}$ & $\mathrm{CHO} / \mathrm{S}$ & NAA/CRE & LIPS/CHO & NAA / S & & & \\
\hline $\begin{array}{l}\text { Healthy vs } \\
\text { Gliomas }\end{array}$ & $\mathrm{NAA} / \mathrm{CHO}$ & $\mathrm{CHO} / \mathrm{CRE}$ & $\mathrm{CHO} / \mathrm{S}$ & LAC/CRE & ALA/CRE & $\mathrm{MI} / \mathrm{S}$ & & & $0.99 / 0.005$ \\
\hline $\begin{array}{c}\text { Healthy vs } \\
\text { MNG }\end{array}$ & $\mathrm{MI} / \mathrm{S}$ & NAA / S & & & & & & & $0.98 / 0.009$ \\
\hline $\begin{array}{c}\text { GR2 vs } \\
\text { GR3 }\end{array}$ & LIPS/CRE & $\mathrm{CRE} / \mathrm{S}$ & LIPS/CHO & $\mathrm{LAC} / \mathrm{CRE}$ & ALA/CRE & $\mathrm{MI} / \mathrm{S}$ & ALA / S & NAA/CRE & $0.84 / 0.028$ \\
\hline $\begin{array}{l}\text { GR2 vs } \\
\text { GR4 }\end{array}$ & $\mathrm{CRE} / \mathrm{S}$ & $\mathrm{CHO} / \mathrm{S}$ & $\mathrm{MI} / \mathrm{S}$ & ALA / S & & & & & $0.99 / 0.004$ \\
\hline $\begin{array}{l}\text { GR3 vs } \\
\text { GR4 }\end{array}$ & $\mathrm{CHO} / \mathrm{S}$ & $\mathrm{MI} / \mathrm{S}$ & & & & & & & $0.98 / 0.013$ \\
\hline $\begin{array}{l}\text { GR2 vs } \\
\text { MNG }\end{array}$ & $\mathrm{NAA} / \mathrm{CHO}$ & ALA/CRE & GLU1/GLU2 & L1/ CRE & NAA/CRE & $\mathrm{MI} / \mathrm{CRE}$ & GLX/CRE & & $0.92 / 0.022$ \\
\hline $\begin{array}{l}\text { GR3 vs } \\
\text { MNG }\end{array}$ & ALA / S & $\mathrm{NAA} / \mathrm{CHO}$ & $\mathrm{MI} / \mathrm{S}$ & $\mathrm{LAC} / \mathrm{CHO}$ & $\mathrm{MI} / \mathrm{CHO}$ & $\mathrm{NAA} / \mathrm{S}$ & $\mathrm{CHO} / \mathrm{S}$ & & $0.94 / 0.015$ \\
\hline $\begin{array}{l}\text { GR4 vs } \\
\text { MNG }\end{array}$ & NAA/CHO & LIPS/CHO & NAA / S & $\mathrm{LAC} / \mathrm{CRE}$ & ALA / S & & & & $0.90 / 0.018$ \\
\hline
\end{tabular}

TABLE II

BEHAVIOR OF THE RATIO MARKERS IN EACH BINARY CLASSIFICATION SCHEME

\begin{tabular}{|c|c|c|c|c|c|c|c|c|c|c|c|c|c|c|c|c|c|c|}
\hline $\begin{array}{c}\text { Binary } \\
\text { schemes }\end{array}$ & $\begin{array}{l}\text { NAA/ } \\
\text { CHO }\end{array}$ & $\begin{array}{c}\text { NAA / } \\
\text { CRE }\end{array}$ & $\begin{array}{c}\text { NAA / } \\
\mathrm{S}\end{array}$ & $\begin{array}{l}\mathrm{CHO} / \\
\mathrm{CRE}\end{array}$ & $\begin{array}{c}\mathrm{CHO} / \\
\mathrm{S}\end{array}$ & $\begin{array}{l}\mathrm{CRE} / \\
\mathrm{S}\end{array}$ & $\begin{array}{l}\mathrm{MI} / \\
\mathrm{CHO}\end{array}$ & $\begin{array}{l}\mathrm{MI} / \\
\mathrm{CRE}\end{array}$ & $\mathrm{MI} / \mathrm{S}$ & $\begin{array}{l}\text { LAC / } \\
\text { CHO }\end{array}$ & $\begin{array}{l}\text { LAC / } \\
\text { CRE }\end{array}$ & $\begin{array}{l}\text { ALA / } \\
\text { CRE }\end{array}$ & $\begin{array}{c}\text { ALA / } \\
\text { S }\end{array}$ & $\begin{array}{l}\text { LIPS / } \\
\text { CHO }\end{array}$ & $\begin{array}{l}\text { LIPS / } \\
\text { CRE }\end{array}$ & $\begin{array}{l}\text { L1/ } \\
\text { CRE }\end{array}$ & $\begin{array}{l}\text { GLU1/ } \\
\text { GLU2 }\end{array}$ & $\begin{array}{c}\text { GLX / } \\
\text { CRE }\end{array}$ \\
\hline $\begin{array}{c}\text { Healthy vs } \\
\text { Tumors }\end{array}$ & $\downarrow \downarrow$ & $\uparrow$ & $\downarrow \downarrow$ & $\uparrow$ & $\uparrow \uparrow$ & & & & & & & & & $\downarrow$ & & & & \\
\hline $\begin{array}{l}\text { Healthy vs } \\
\text { Gliomas }\end{array}$ & $\downarrow \downarrow$ & & & $\uparrow$ & $\uparrow \uparrow$ & & & & $\downarrow \downarrow$ & & $\uparrow$ & $\uparrow$ & & & & & & \\
\hline $\begin{array}{l}\text { Healthy vs } \\
\text { MNG }\end{array}$ & & & $\downarrow$ & & & & & & $\downarrow \downarrow$ & & & & & & & & & \\
\hline GR2 vs GR3 & & $\uparrow$ & & & & $\downarrow \downarrow$ & & & $\downarrow$ & & $\uparrow \uparrow$ & $4 \uparrow$ & $\uparrow \uparrow$ & $\uparrow$ & $\uparrow \uparrow$ & & & \\
\hline GR2 vs GR4 & & & & & $\downarrow \downarrow$ & $\downarrow \downarrow$ & & & $\downarrow \downarrow$ & & & & $\uparrow \uparrow$ & & & & & \\
\hline GR3 vs GR4 & & & & & $\downarrow \downarrow$ & & & & $\downarrow \downarrow$ & & & & & & & & & \\
\hline GR2 vs MNG & $\uparrow$ & $\downarrow \downarrow$ & & & & & & $\downarrow$ & & & & $4 \uparrow$ & & & & $\uparrow$ & $\downarrow$ & $\uparrow$ \\
\hline GR3 vs MNG & $\downarrow$ & & $\downarrow \downarrow$ & & $\uparrow \uparrow$ & & $\downarrow$ & & $\uparrow$ & $\uparrow$ & & & $\uparrow \uparrow$ & & & & & \\
\hline GR4 vs MNG & $\uparrow$ & & $\downarrow \downarrow$ & & & & & & & & $\uparrow$ & & $\uparrow$ & $\uparrow$ & & & & \\
\hline
\end{tabular}

Single arrow corresponds to a significant change $(\mathrm{p}<0.05)$ in the mean of the ratio marker in the second class, while double arrow reflects a highly significant change $(\mathrm{p}<0.001)$. Upwards direction corresponds to an increase, downwards direction to a decrease in the mean.

\section{DISCUSSION}

Type and grade discrimination of the tumors can be revealed by tracking the trends in the behavior of the ratio markers in each class, as presented in Table II. We can observe that the 9 classification schemes can be merged to 3 major groups, which are studied next.

\section{A. Discrimination of Healthy tissue from Tumors}

Healthy vs Tumorous tissues discrimination can be achieved by examining the 3 NAA ratios, the 2 CHO ratios and the LIPS/CHO ratio. It can be observed that NAA/CHO,
$\mathrm{NAA} / \mathrm{S}$ and $\mathrm{CHO} / \mathrm{S}$ are highly significant markers. This also reveals that high grade tumors are contained in the Tumors class due to the fact that NAA is highly decreased in high grade tumors like GR4 and that $\mathrm{CHO}$ and LIPS are also increased in Gliomas and in some cases of Meningiomas [9].

Discrimination of Healthy vs Gliomas can be obtained by examining the $\mathrm{NAA} / \mathrm{CHO}$ ratio, the $2 \mathrm{CHO}$ ratios and the $\mathrm{MI} / \mathrm{S}, \mathrm{LAC} / \mathrm{CRE}$ and ALA/CRE ratios. As already mentioned, $\mathrm{NAA} / \mathrm{CHO}$ and $\mathrm{CHO} / \mathrm{S}$ ratios are expected to be highly significant markers in this case. $\mathrm{MI} / \mathrm{S}$ ratio is also highly significant due to the fact that MI is high in Low 
Grade Gliomas like GR2 and some cases of GR3 [10] but low in GR4. Even more LAC is increased, CRE is decreased and sometimes ALA observed increased in Gliomas [9].

Healthy tissues can be distinguished from MNG by examining the NAA/S and the MI/S ratios. MI/S is highly significant due to the fact that $\mathrm{MI}$ is almost absent in Meningiomas but not in Healthy tissue [11]. Also NAA is expected to be decreased in MNG [9].

\section{B. Discrimination of Gliomas}

Discrimination of Gliomas Grade II (GR2) from Gliomas Grade III (GR3) can be obtained by taking under consideration the ratios NAA/CRE, $\mathrm{CRE} / \mathrm{S}, \mathrm{MI} / \mathrm{S}$, LAC/CRE but also the 2 ALA and 2 LIPS ratios. The highly significant ratios of CRE/S, LAC/CRE, ALA/CRE, ALA/S and the LIPS/CRE reveal the effects of CRE, which is reduced in High Grade Gliomas like GR3, but also the metabolic behaviour of LIPS, LAC and sometimes ALA that are increased in these tumors [11]. For discriminating Gliomas Grade II (GR2) from the Highest Grade Gliomas (GR4), also called Glioblastomas, four highly significant ratios have to be evaluated. These are the $\mathrm{CHO} / \mathrm{S}, \mathrm{CRE} / \mathrm{S}$, $\mathrm{MI} / \mathrm{S}$ and ALA/S ratios. As already mentioned, as tumor grade increases the CRE is decreased and the $\mathrm{CHO}$ is elevated. MI, as mentioned, is also increased in Low Grade Gliomas (GR2) but is low in GR4. Gliomas Grade III and Grade IV, usually have metabolic similarities due to the fact that they are often observed in necrotic areas, with increased lipids and lactate. This fact reflects the importance of $\mathrm{CHO} / \mathrm{S}$ and $\mathrm{MI} / \mathrm{S}$ ratios, which are able to distinguish the two tumor types, due to greater increase of $\mathrm{CHO}$ and decrease of MI in Gliomas Grade IV.

\section{Discrimination of Gliomas from Meningiomas}

Gliomas and Meningiomas are originated in different areas of brain. ALA is increased in Meningiomas [12]. So, both Low Grade Gliomas (GR2), intermediate (GR3) and High Grade Gliomas (GR4) can be differentiated from Meningiomas using the ratios ALA/CRE and ALA/S. As Table II indicates, however, it seems that L1 [13] and GLX [14] play an important role in GR2 vs Meningiomas similar to the NAA/CRE ratio, since NAA is more reduced in Meningiomas than GR2 [9]. Other significant ratio marker that also contribute is the MI/S, since MI is almost absent in Meningiomas [11].

It is worth emphasizing that Table I includes those features that provide the best AUROC measure per classification scheme. Thus, all other ratio-features were discarded by the feature selection process as not of high importance for the discrimination of tumors. In other words, discarded features had no additional value in accuracy. This study, in contrast to others [2], [3] involving ratio-type features to discriminate brain tumors, aims at revealing the smallest possible set of ratio features that can successfully classify a new subject to one of the classes considered. For this purpose, the discriminative potential of new ratio features, which have not been considered before, was extensively studied.

\section{V.CONCLUSIONS}

It becomes widely accepted that metabolomics is a highly promising field that will definitely accelerate the discovery of individualised therapies for brain cancer. Along these directions, the processing of the metabolite spectrum (area under the peaks and the ratios of metabolite areas under the peaks) has derived features that enable the effective classification of several types of brain tumors. Advanced feature selection methods further enable the specification of ratio combinations that allow for high discriminative power.

\section{ACKNOWLEDGMENT}

Present work was supported by Biopattern, IST EU funded project, Proposal/Contract no.: 508803.

\section{REFERENCES}

[1] H. Kugel et al., "Human brain tumors: spectral patterns detected with localised H-1 MR spectroscopy", Radiology, vol. 183, pp.701-709, June 1992.

[2] M. F. Chernov, M. Hayashi, M. Izawa, Y. Ono and T. Hori, "Proton magnetic resonance spectroscopy (MRS) of metastatic brain tumors: variations of metabolic profile", International Journal of Clinical Oncology, vol. 11, pp. 375-384, October 2006.

[3] F. G. Lehnhardt, C. Bock, G. Rohn, R. Ernestus and M. Hoehn, "Metabolic differences between primary and recurrent human brain tumors: a ${ }^{1} \mathrm{H}$ NMR spectroscopic investigation", $N M R$ in Biomedicine,vol. 18, pp. 371-382, May 2005.

[4] A.W. Simonetti et al., "A chemometric approach for brain tumor classification using magnetic resonance imaging and spectroscopy", Analytical Chemistry, vol. 75, pp. 5352-5361, September 2003.

[5] F. A. Howe and K. S. Opstad, "H MR spectroscopy of brain tumors and masses", NMR in Biomedicine, vol. 16, pp. 123-131, April 2003.

[6] M. E. Blazadonakis and M. Zervakis, "Polynomial and RBF kernels as marker selection tools - A breast cancer case study", in Proceedings of the 6th International Conference on Machine Learning and Applications, Cincinnati Ohio, 2007, pp. 488-493.

[7] N. V. Vapnik, The Nature of Statistical Learning Theory New York: Springer-Verlag, 1999.

[8] J. A. K. Suykens and J. Vandewalle, "Least squares support vector machine classifiers", Neural Processing Letters, vol. 9, pp. 293-300, June 1999.

[9] C. Majós et al., "Adult primitive neuroectodermal tumor: proton MR spectroscopic findings with possible application for differential diagnosis", Radiology, vol. 225, pp. 556-566, September 2002.

[10] M. Castillo, J. K. Smith and L. Kwock, "Correlation of myo-inositol levels and grading of cerebral astrocytomas", American Journal of Neuroradiology, vol. 21, pp. 1645-1649, October 2000.

[11] V. Callot et al., "1 H MR spectroscopy of human brain tumors: a practical approach", European Journal of Radiology, vol. 67, pp. 268-274, August 2008.

[12] A. Heerschap, "In vivo magnetic resonance spectroscopy in clinical oncology", in Cancer Drug discovery and Development In Vivo Imaging of Cancer Therapy, A. F. Shields and P. Price, Ed. Totowa NJ: Human Press Inc, 2007, pp. 241-258.

[13] Q. Gang et al., "Lipid signal in evaluation of intracranial meningiomas", Chinese Medical Journal, vol. 121, pp. 2415-2419, December 2008.

[14] S. Hazany, J. R. Hesselink, J. F. Healy and S. G. Imbesi, "Utilization of glutamate/creatine ratios for proton spectroscopic diagnosis of meningiomas", Neuroradiology, vol. 49, pp. 121-127, February 2007. 